\title{
Coarse and fine-tuning of lasing transverse electromagnetic modes in coupled all-inorganic perovskite quantum dots
}

\author{
Youngsin Park ${ }^{1, \S}$, Guanhua Ying ${ }^{2, \S}$, Atanu Jana ${ }^{1, \S}$, Vitaly Osokin ${ }^{2}$, Claudius C. Kocher ${ }^{2}$, Tristan Farrow ${ }^{3,2}(\bowtie)$, \\ Robert A. Taylor ${ }^{2}(\bowtie)$, and Kwang S. $\operatorname{Kim}^{1}(\square)$ \\ ${ }^{1}$ School of Natural Science, Ulsan National Institute of Science and Technologh, Ulsan 44919, Republic of Korea \\ ${ }^{2}$ Clarendon Laboratory, Department of Physics, University of Oxford, Parks Road, Oxford, OX1 3PU, UK \\ ${ }^{3}$ Centre for Quantum Technologies, National University of Singapore, Science Drive 2, Singapore 117543, Singapore \\ ${ }^{\S}$ Youngsin Park, Guanhua Ying, and Atanu Jana contributed equally to this work.
}

(C) The Author(s) 2020

Received: 20 May 2020 / Revised: 10 August 2020 / Accepted: 11 August 2020

\begin{abstract}
Inorganic perovskite lasers are of particular interest, with much recent work focusing on Fabry-Pérot cavity-forming nanowires. We demonstrate the direct observation of lasing from transverse electromagnetic (TEM) modes with a long coherence time $\sim 9.5$ ps in coupled $\mathrm{CsPbBr}_{3}$ quantum dots, which dispense with an external cavity resonator and show how the wavelength of the modes can be controlled via two independent tuning-mechanisms. Controlling the pump power allowed us to fine-tune the TEM mode structure to the emission wavelength, thus providing a degree of control over the properties of the lasing signal. The temperature-tuning provided an additional degree of control over the wavelength of the lasing peak, importantly, maintained a constant full width at half maximum (FWHM) over the entire tuning range without mode-hopping.
\end{abstract}

\section{KEYWORDS}

perovskite quantum dots, coherent lasing, transverse electromagnetic mode, stimulated emission

\section{Introduction}

All-inorganic lead halide perovskites such as alkali-metal caesium lead halides $\left(\mathrm{CsPbX}_{3}, \mathrm{X}=\mathrm{Cl}, \mathrm{Br}\right.$ or $\left.\mathrm{I}\right)$ are emerging as highly promising materials for optoelectronic devices, from solar cells to light emitting diodes, due to their higher stability and robustness compared to organic-inorganic lead halides [1-8]. Lasing has so far been demonstrated in $\mathrm{CsPbr}_{3}$ nanowires (NWs) with Fabry-Pérot cavity modes bounded on the either end by the facets of single-crystal NWs [7-10] whose diameter needs to exceed $\sim 180 \mathrm{~nm}$ to confine efficiently emission at a wavelength $\sim 535 \mathrm{~nm}$ [7] and to increase the spectral coherence of laser devices. A key factor is the narrowness of the emission line measured at the full width at half maximum (FWHM). It is noteworthy that lasing from $\mathrm{CsPbBr}_{3}$ arises from self-assembled clusters of nanocrystals that encapsulate the quantum dots (QDs) without reliance on an external cavity resonator, unlike the confinement provided by their singlecrystal nanowire counterparts. The nanocrystals are distributed homogeneously with a spacing $\sim 20-30 \mathrm{~nm}$ between each nanocrystal inside a self-assembled cluster characterized by a regular cuboidal geometry with facets $\sim 1-2 \mu \mathrm{m}$ in diameter (Fig. 1(a)). The QDs were synthesized by solution-based processing (see the Electronic Supplementary Material (ESM) for Chemicals and Synthesis) and show a broad spectral linewidth of $\sim 5 \mathrm{~nm}[11,12]$. Although an improved spectral linewidth of $\sim 2 \mathrm{~nm}$ can be achieved by embedding colloidal quantum dots into silica spherical cavities, this approach is not suitable for practical applications [11].

Here we present the first observation of lasing from transverse electromagnetic (TEM) modes in $\mathrm{CsPbBr}_{3}$ QD clusters, generated by stimulated emission (SE) from single excitons and characterized by a coherence time of the lasing signal $\sim 9.5$ ps. We demonstrate also how the wavelength of lasing mode can be continuously controlled without degrading the signal quality using two independent mechanisms, including optical pump power and temperature-tuning. By varying the optical pump power, we show how the signal can be finetuned across a fixed frequency comb of TEM modes, whereas by controlling the temperature, we show coarse-tuning of the lasing signal while maintaining a constant FWHM and avoiding mode-hopping.

\section{Experimental}

\subsection{Sample preparation}

High-quality $\mathrm{CsPbBr} 3$ QDs were synthesized using a top-down fabrication approach which is easy to reproduce and highly cost-effective. First, we synthesized the bulk $\mathrm{CsPbr}_{3}$ by dissolving $\mathrm{PbBr}_{2}$ in hydrobromic acid and then subsequent addition of $\mathrm{Cs}_{2} \mathrm{CO}_{3}$ gives the yellowish orange precipitate. The bulk product and octylammonium bromide were dissolved in dimethylformamide (DMF) and then toluene was added to it resulting in the yellow colloidal solution. Octylammonium acts as capping ligand to arrest the precipitation of the $\mathrm{CsPbBr}_{3}$ QDs. 
(a)

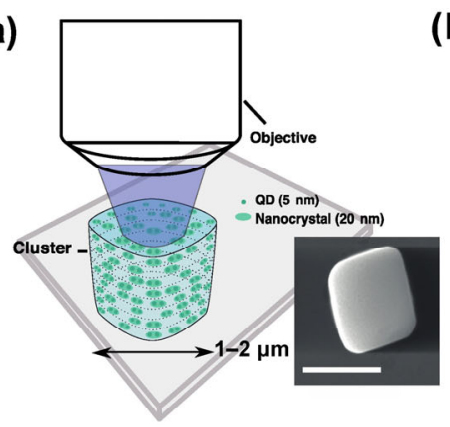

(d)

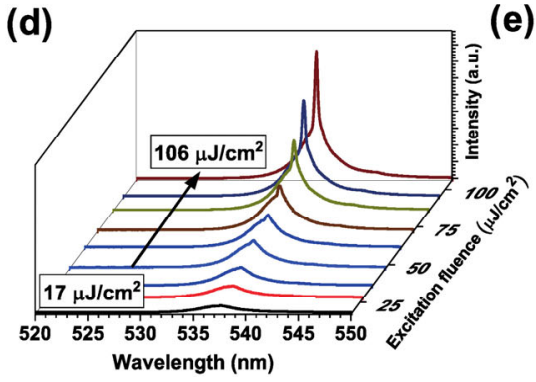

(b)

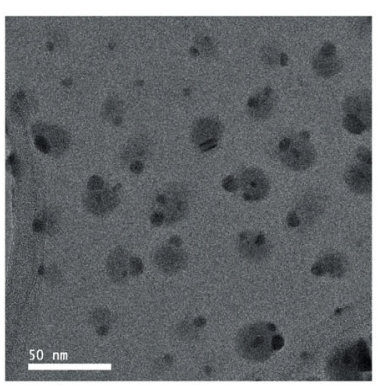

(c)

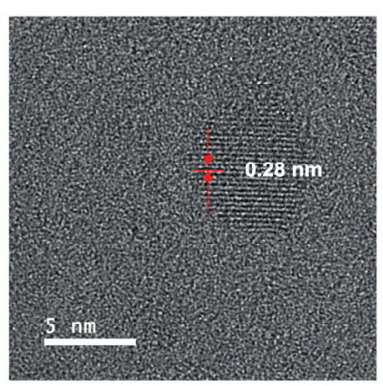

(e)

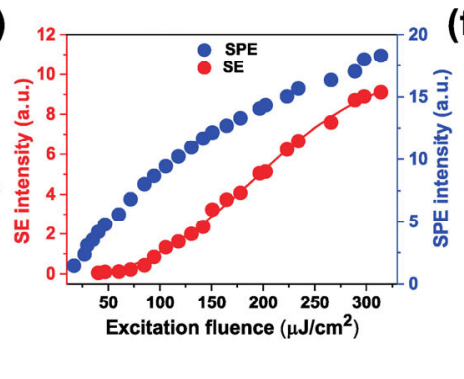

(f)

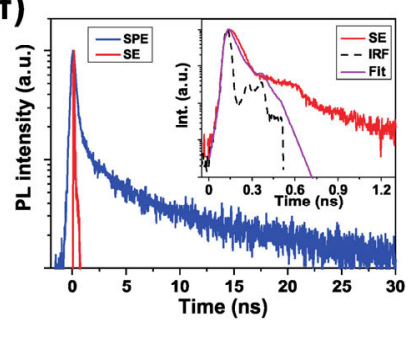

Figure 1 (a) Schematic of a self-assembled cluster of nanocrystals. Each nanocrystal comprises two to three $\mathrm{CsPbBr} 3 \mathrm{QDs}_{3}$ The side of the cluster ranges between $\sim 1-2 \mu \mathrm{m}$, compared to the much smaller nanocrystals $\sim 20 \mathrm{~nm}$ in diameter. The laser excitation area illuminates hundreds of nanocrystals. Bottom right is an SEM image of a cluster (scale bar is $1 \mu \mathrm{m}$ ). (b) Transmission electron microscope image of CsPbBr 3 QDs inside a cluster. The dark spots inside the clusters are individual QDs (diameter of $\sim 5 \mathrm{~nm}$ ). (c) High-resolution transition electron microscope image of a QD, highlighting the crystallinity of the assembly with a lattice spacing of $0.28 \mathrm{~nm}$. (d) Power-dependent PL spectra at a low excitation fluence regime below $\sim 106 \mu \mathrm{J} / \mathrm{cm}^{2}$. Each spectrum shows a broad background SPE with a FWHM of $\sim 3 \mathrm{~nm}$ due to the ensemble effect. (e) Integrated emission intensity of the background SPE and the sharp lasing peak (with the SPE subtracted) as a function of excitation power. An S-shape characteristic of lasing can be seen for the SE trace, with an onset and saturation, while the intensity of the SPE emission continues to increase linearly. (f) Time resolved photoluminescence at $\sim 200 \mu \mathrm{J} / \mathrm{cm}^{2}$ for the SE and SPE signals at a low excitation fluence $\sim 95 \mathrm{~nJ} / \mathrm{cm}^{2}$, with the inset detailing the same decay within the first nanosecond.

The nanoparticles were separated by a centrifuge operating at $5,000 \mathrm{rpm}$ for $5 \mathrm{~min}$.

\subsection{Structural characterization}

Powder X-ray diffraction was measured on a D/MAX2500V/PC diffractometer, Rigaku using a $\mathrm{Cu}$-rotating anode $\mathrm{X}$-ray source. The Bragg diffraction angle $(2 \theta)$ range was set to $10^{\circ}-50^{\circ}$ and the scan rate was $2 \%$ min. Transmission electron microscopy images were taken on a JEOL JEM-2100F electron microscope using a $200 \mathrm{kV}$ electron source.

\subsection{Photoluminescence measurements}

A 70 ps pulsed diode laser operating at $450 \mathrm{~nm}(20 \mathrm{MHz}$ repetition rate) and a frequency-doubled femtosecond Ti:sapphire laser (100 fs pulses at $76 \mathrm{MHz}$ ) operating at 400 $\mathrm{nm}$ were used to excite the $\mathrm{CsPBr}_{3}$ in the $\mu$-PL experiments. The sample was mounted in a continuous-flow helium cryostat. A $100 \times$ reflecting objective was held by a sub-micron precision piezoelectric stage and used to focus the laser to a spot size of $\sim 1 \mu \mathrm{m}$ and to collect the resulting luminescence. The luminescence was then directed to a spectrometer (spectral resolution of $0.15 \mathrm{~nm}$ ) confocally via a multi-mode optical fiber of $25 \mu \mathrm{m}$ core size to limit the collection area to a spot of $\sim$ $0.4 \mu \mathrm{m}$. For coherence measurement, a Michelson interferometer was built with a non-polarizing 50:50 beamsplitter for directing the PL signal into the two arms. A cage translator with $1 / 2$ " $z$-axis travel micrometer of 0.001 " per graduation precision was deployed as a delay stage. The interference pattern was then directed into a camera for real-time adjustment. Time resolved photoluminescence (TRPL) measurements were carried out using the same experimental set up as above. The dispersed $\mathrm{PL}$ was reflected towards a photomultiplier connected to a commercial photon counting system (Becker\&Hickl SPC-130), with a time resolution of $\sim 35$ ps.

\section{Results and discussion}

The crystal structure of the $\mathrm{CsPBr}_{3}$ QDs was characterized by X-ray diffraction (see Fig. S1 in the ESM), revealing orthorhombic or cubic symmetries [13-16]. Images of individual $\mathrm{CsPbBr}_{3}$ QDs obtained by transmission electron microscopy (Figs. 1(a) and 1(b)) show clear lattice fringes and the interplanar distance is estimated to be $\sim 0.28 \mathrm{~nm}$, in good agreement with that of the (200) plane of a $\mathrm{CsPBr}_{3}$ crystal with diameters $\sim 3.5-7 \mathrm{~nm}[13,17]$. The morphology of the QDs is consistent with that reported elsewhere, such as in Di et al. [17], and should not be confused with lead precipitates that can result from transmission electron microscopy beam damage to the sample [18]. Individual QDs deposited on a copper substrate are presented in Fig. S2 in the ESM under transmission electron microscopy. On average, two or three QDs are encapsulated in a so-called nanocrystal with dimensions $\sim 20 \mathrm{~nm}$ (Fig. S2 in the ESM). In turn, the nanocrystals are distributed homogeneously with a spacing $\sim 20-30 \mathrm{~nm}$ inside a self-assembled cluster characterized by a regular geometry with facets $\sim 1-2 \mu \mathrm{m}$ in diameter (Fig. 1).

Power-dependent photoluminescence (PL) measurements were performed on individual clusters with diameters $\sim 2 \mu \mathrm{m}$ with the spectra showing excitonic PL by spontaneous emission (SPE) in the low excitation fluence regime (Fig. 1(d)), whilst at high excitation fluences very sharp and intense SE peaks emerge at the onset of lasing (Fig. S3 in the ESM). Direct measurement of the lasing signal yielded a characteristic $\mathrm{S}$-shaped intensity curve (Fig. 1(e)) showing an onset threshold $\left(P_{\text {th }}\right)$ at a fluence $\sim 75 \mu \mathrm{J} / \mathrm{cm}^{2}$ and a saturation intensity of $\sim 300 \mu \mathrm{J} / \mathrm{cm}^{2}$. Lasing was corroborated by TRPL, which shows that the lifetime of the SE is three orders of magnitude shorter than that of the SPE [19]. The decay curve for the SE signal (inset in Fig. 1(f)) yields a time constant of $\sim 45$ ps that is 
much faster than the SPE, and is consistent with a fast lasing lifetime [19]. The decay curve for SPE shows a biexponential decay with lifetimes of 310 ps and 5.96 ns for the fast and slow components respectively. The fast component agrees with value measured previously in $\mathrm{CsPbBr}_{3}$ nanocrystals [20].

A sub-threshold PL spectrum taken below an excitation power of $\sim 45 \mu \mathrm{J} / \mathrm{cm}^{2}$ shows a FWHM of $\sim 0.085 \mathrm{~nm}$ for the SE peak (Fig. S4 in the ESM). As the peak width is limited by the resolution from our spectrometer $(0.15 \mathrm{~nm})$, the actual width is smaller, presenting one of the smallest recorded values for $\mathrm{CsPbr}_{3}$ single crystals $[4,6,9]$. We note further that for a small number of clusters two peaks are present, Figs. 2(a) and 2(b), which match the energy of those reported elsewhere [21], attributed to emission from coupled and uncoupled QDs. The SE peak emerges at energies near $536 \mathrm{~nm}$, as expected for emission from an ensemble of coupled QDs with nearidentical structures. This is further underscored by the highly reproducible red-shifted emission line (Fig. 2(b)). The coherence time of the lasing emission was measured using a Michelson interferometer, yielding a coherence time of $\sim 9.5$ ps for the SE peak (Figs. 2(c) and 2(d)). That is two orders longer than the SPE coherence time of $41 \mathrm{fs}$ obtained from a Gaussian fit and provides clear evidence that the SE originates from a spectrally coherent narrow mode that is lasing as opposed to incoherent amplified spontaneous emission (ASE).

Generally, wave guiding in QDs without an external cavity can be discounted due to their small size compared to the emission wavelength. Nevertheless, our QD clusters behave as a de-facto optical cavity if we consider that the walls of the clusters form an intrinsic resonator. This is surprising considering that the clusters are not single crystals, unlike nanowires that form facets naturally. Lasing spectra obtained by confocal imaging integrating over an area above the center of a typical cluster reveals TEM modes with circular symmetric spatial mode profiles, represented (Fig. S5 in the ESM) as surface intensity plots for individual mode wavelengths.

Circularly symmetric beam profiles can be described by Gaussian modes under the paraxial wave approximation [22]. Considering the cuboidal shape of our QD clusters we choose planar symmetric Gauss-Hermite modes (Eq. (1)). We note that only the phase terms, rather that spatial terms or the actual cavity geometry, are necessary for estimating the mode spacing.

$$
\begin{aligned}
& E_{m, n}^{\mathrm{H}}= \\
& \frac{1}{w(\zeta)} H_{m}\left[\sqrt{2} \frac{x}{\mathrm{w}(\zeta)}\right] H_{n}\left[\sqrt{2} \frac{x}{\mathrm{w}(\zeta)}\right] \exp \left[\mathrm{i} k z-\frac{\rho^{2}}{w_{0}^{2}(1+\mathrm{i} \zeta)}-\mathrm{i} \psi_{m, n}\right]
\end{aligned}
$$

The symbols in the equation follow the nomenclature in [22]. For a given longitudinal mode supported by a cavity, the phase term determines the frequency of the transverse modes. Hence the frequencies of the transverse modes can be characterized by:

$$
f_{i, j, z}^{\mathrm{H}}=\frac{c}{2 L n}\left[z+(j+2 i+1) \frac{2 \arctan \left(\frac{\sqrt{1-g_{1} g_{2}}}{1+g_{1} g_{2}}\right)}{\pi}\right]
$$

where $i$ and $j$ are integers, $n$ is the refractive index of the gain medium, $L$ is the size of the cavity, $z$ is the fundamental Gaussian mode index, and are the parameters describing the curvature of the cavity surfaces given by $g_{i}=1-L_{i} / \beta_{i}$, with $\beta$ the curvature factor. With our cluster radius $\sim 1 \mu \mathrm{m}$ and refractive index $\sim 2.2-2.3$ [6], the modal wavelength separation can be as low as $1 \mathrm{~nm}$ between a higher order TEM mode and a fundamental mode with a higher Gaussian mode index. Conversely, the same two-dimensional (2D) maps for the SPE show no mode structure (Fig. S6 in the ESM).

The dependence of the TEM mode structure was characterized as a function of the optical pump power, as shown in Fig. 3(a) highlighting the mode profiles at increasing powers obtained from a single QD cluster. As the excitation fluence increases, new fundamental modes are occupied to the higher energy (blue) side, Fig. 3(b), whilst the lower-energy (red) ones fade and higher order TEM modes are populated as the underlying emission blueshifts. This process arises as the PL emission populates the fixed comb of optical cavity modes by coupling to the fixed transverse modes of the cavity (outlined by boxes in Fig. 4(b)).
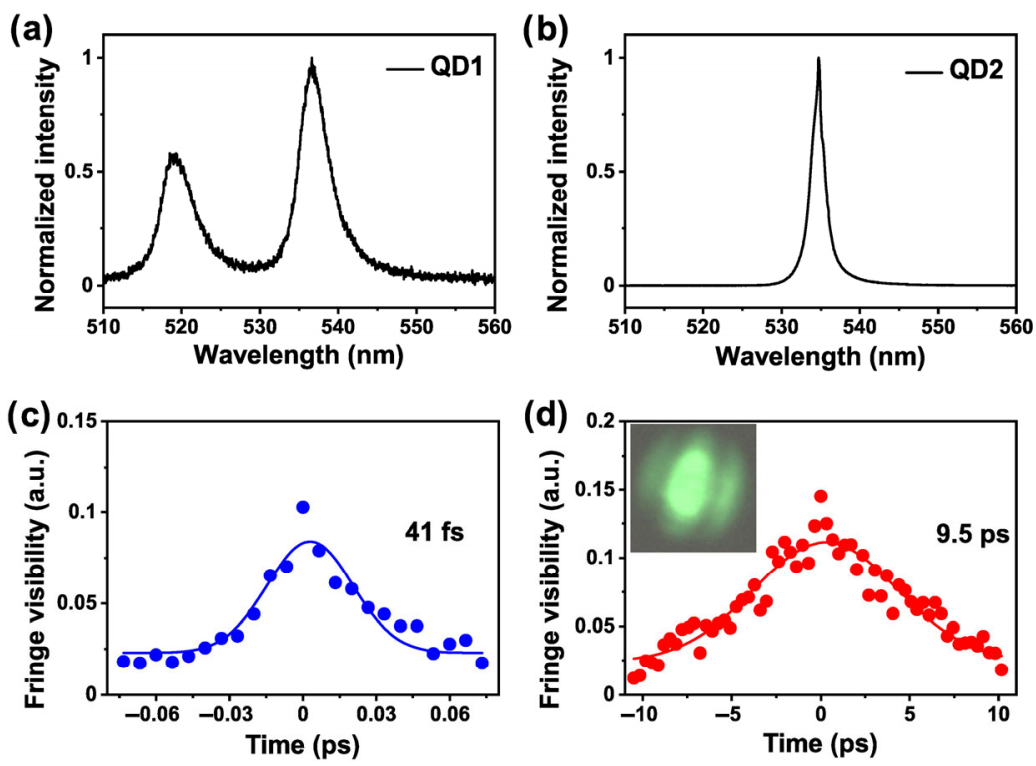

Figure 2 (a) The longer wavelength emission corresponds to SE from QD clusters. (b) Short-wavelength emission is from isolated or uncoupled QDs. (c) and (d) First-order correlation of the SPE and SE emission obtained from the interference fringe visibility resulting from the overlap of the emission signal in the two arms of a Michelson interferometer (inset in (d) shows the real fringe image at zero time-delay). The fitted correlation function of both the SE and SPE are presented, with the SE peak showing an exponential decay with a long coherence time of 9.5 ps corresponding to a linewidth of $105 \mathrm{GHz}$. The solid lines are Gaussian fits of the data. 
Mode-tuning by controlling the optical pump power provides a degree of control over the properties of the lasing signal. We repeated the test on several clusters to corroborate the results, thus confirming that the effect is reproducible (Figs. 4(a) and 4(b) and Fig. S7 in the ESM). For our low pump fluences we calculated that the maximum possible temperature rise would be of the order $\sim 1 \mathrm{~K}$. Given that a $1 \mathrm{~nm}$ blueshift of the emission would require a temperature rise of $\sim 10 \mathrm{~K}$ (Fig. 4 (d)) taken from another cluster where only single mode is supported), we conclude that the blueshift of the background has a different origin to that resulting typically from a heat-induced phase transition. Pump-probe PL studies of the dynamics of hot carriers during thermalization and cooling phases suggest that the blueshift in lead halides originates from band-filling of the density of states by energetic carriers [23, 24]. In the immediate aftermath of photoexcitation, carriers whose temperature exceeds the crystal temperature thermalize by scattering with other carriers and optical phonons. This is followed by a slower cooling phase mediated by carrier-phonon scattering when the radiative recombination between carriers away from the band-edge, where they form a Boltzmann energy distribution, dominates. This becomes apparent in the net blueshift of our time-integrated PL spectra as the emission shifts to higher energy. As the pump fluence increases, the density of states occupied by carriers increases thus filling the bands to higher energy, resulting in a further blueshift of the (a)

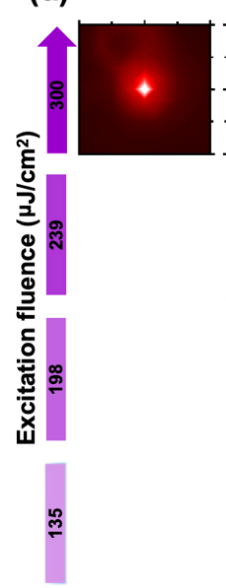

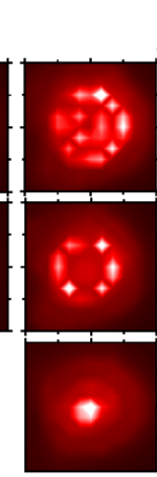

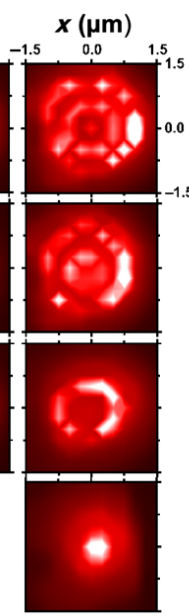

Stimulated mode wavelength $(\mathrm{nm})$

$\begin{array}{llll}\mathbf{5 3 3 . 0 9 5} & \mathbf{5 3 4 . 0 5 3} & \mathbf{5 3 4 . 8 4 5} & \mathbf{5 3 5 . 6 7 5}\end{array}$

(b)

Wavelength $(\mathrm{nm})$

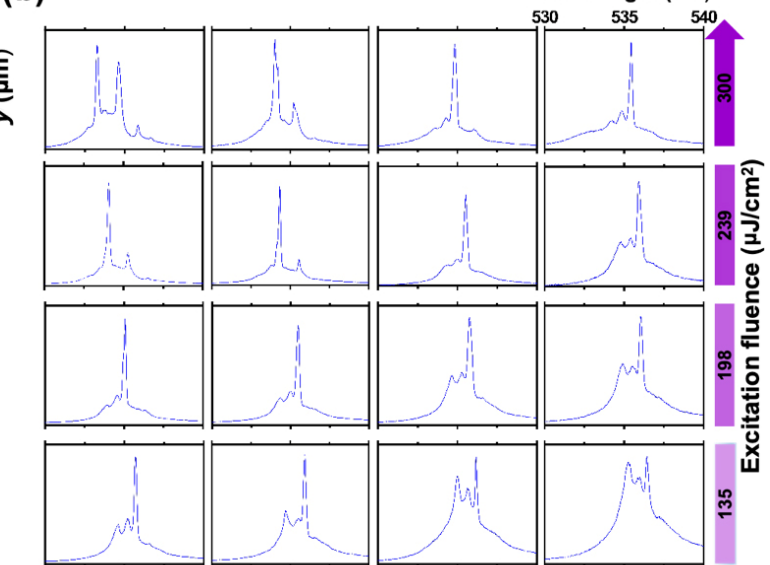

Figure 3 (a) Fluence-dependent PL 2D maps for an area $3 \mu \mathrm{m} \times 3 \mu \mathrm{m}$ showing the intensity distribution in the beam cross-section of TEM lasing modes at different fixed wavelengths emitted by a single $\mathrm{CsPBr}_{3} \mathrm{QD}$ cluster. With increasing fluence, higher-order TEM modes are occupied at higher energy and lower-energy modes fade as the underlying emission blueshifts. The same behavior is observed in different clusters. (b) Power-dependent PL spectra taken at in an increasing radial distance from the center of the QD cluster.

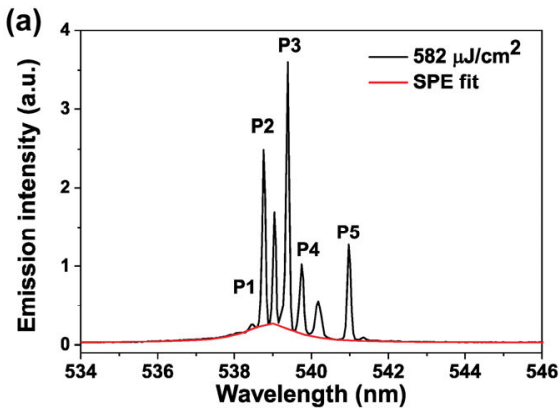

(b)

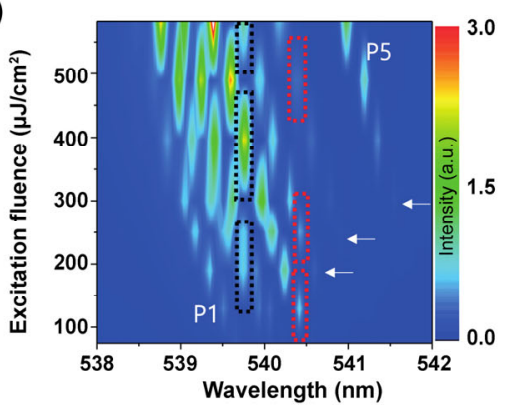

(c)
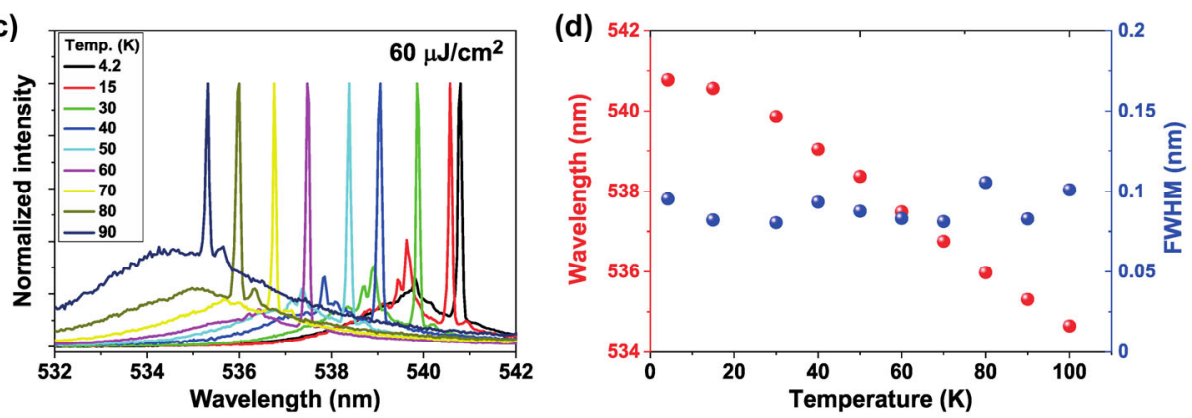

Figure 4 (a) Emission spectrum at excitation fluence of $\sim 582 \mu \mathrm{J} / \mathrm{cm}^{2}$ showing intense and sharp peaks (marked P1 to P5) on top of a background emission around $539 \mathrm{~nm}$ arising from excitonic recombination. At higher excitation powers the wavelength of the sharp lines shifts to bluer wavelength, while additional peaks emerge (Fig. S7 in the ESM) and become dominant. The average FWHM of each peak is $\sim 0.1 \mathrm{~nm}$. (b) Power-dependent $2 \mathrm{D}$ contour map of emission spectra taken over a region of a QD cluster. The black and red rectangles are visual guides showing lasing modes at fixed wavelengths. The white arrows indicate the onset of new peaks. The series of additional peaks that appear with increasing excitation power, from P1 to P5. (c) Temperature-dependent PL tuning of SE2 peak. (d) Wavelength and FWHM of the SE2 peak as a function of temperature. The dashed line provides an eye-guide to highlight that the FWHM of the lasing peak remained almost unchanged by the temperature increase. 
emission. Conversely, as the carriers recombine and deplete the band, the emission energy redshifts, although this was not observed within our optical pumping scheme but has been reported elsewhere [24].

We verified that the blueshift is reproducible across a range of nanocrystals in the sample. The shift is clearly observable even at $20 \mathrm{~K}$ while the temperature difference required for the mode wavelength change in Fig. 4(b) from a thermal effect is $\sim 160 \mathrm{~K}$. Again, this rules out the possibility of heat-induced shift of the PL in Fig. 4(b) where the modes remain at fixed wavelengths. From a technological perspective it is worth noting that the wavelength-tuning is achieved in a controlled manner as it moves between consecutive modes and that the width of the SE peak remains constant within our spectrometer resolution of $0.15 \mathrm{~nm}$ (Fig. 4(d)). It is also worth pointing out that even if the SE peak broadens slightly with temperature, its linewidth remains resolution limited. The two control methods, through optical pump power in tandem with temperature, enable us to coarse- and fine-tune which of the fixed comb of lasing modes is populated whilst maintaining a high-quality coherent signal. While a study of the underlying causes of this effect go beyond the scope of this investigation, future work could focus on carrier dynamics as a function of temperature. By contrast, the amplified spontaneous emission (ASE) from $\mathrm{MAPbI}_{3}$ films red-shifts with power [25, 26], arising from reabsorption [27] or biexciton recombination that is red-shifted by $\sim 10 \mathrm{~nm}$ with respect to the single exciton emission $[28,29]$. The binding energy of biexcitons in $\mathrm{CsPbr}_{3}$ is $38-60 \mathrm{meV}[11,29]$ so we can rule out lasing from biexcitonic recombination.

\section{Conclusion}

In conclusion, we have made a direct observation of TEM lasing modes from clusters of $\mathrm{CsPbBr}_{3}$ QDs and have demonstrated that the modes could be tuned without modehopping by varying the optical pump power, which provided fine-tuning, as well as the temperature for coarse-tuning. Importantly, we showed that the quality of the lasing signal was maintained throughout this process, thereby showcasing the technological potential of inorganic perovskite QDs for deployment in highly coherent tunable micro-lasers in the visible-range and more broadly in ultrafast optoelectronics.

\section{Acknowledgements}

This work was supported by Basic Science Research Program and National Honor Scientist Program through the National Research Foundation of Korea (NRF) (Nos. 2010-0020414 and 2018R1D1A1B07043676). T. F. acknowledges support from the Centre for Quantum Technologies, National University of Singapore.

Electronic Supplementary Material: Supplementary material (TEM images, XRD, and PL intensity variation as a function of temperature data) is available in the online version of this article at https://doi.org/10.1007/s12274-020-3051-y .

Open Access This article is licensed under a Creative Commons Attribution 4.0 International License, which permits use, sharing, adaptation, distribution and reproduction in any medium or format, as long as you give appropriate credit to the original author(s) and the source, provide a link to the Creative Commons licence, and indicate if changes were made.

The images or other third party material in this article are included in the article's Creative Commons licence, unless indicated otherwise in a credit line to the material. If material is not included in the article's Creative Commons licence and your intended use is not permitted by statutory regulation or exceeds the permitted use, you will need to obtain permission directly from the copyright holder.

To view a copy of this licence, visit http://creativecommons.org/licenses/by/4.0/.

\section{References}

[1] Liu, M. Z.; Johnston, M. B.; Snaith, H. J. Efficient planar heterojunction perovskite solar cells by vapour deposition. Nature 2013, 501, 395-398.

[2] Cho, H.; Jeong, S. H.; Park, M. H.; Kim, Y. H.; Wolf, C.; Lee, C. L.; Heo, J. H.; Sadhanala, A.; Myoung, N.; Yoo, S. et al. Overcoming the electroluminescence efficiency limitations of perovskite light-emitting diodes. Science 2015, 350, 1222-1225.

[3] Green, M. A.; Ho-Baillie, A.; Snaith, H. J. The emergence of perovskite solar cells. Nat. Photonics 2014, 8, 506-514.

[4] Møller, C. K. Crystal structure and photoconductivity of cæsium plumbohalides. Nature 1958, 182, 1436.

[5] Lee, M. M.; Teuscher, J.; Miyasaka, T.; Murakami, T. N.; Snaith, H. J. Efficient hybrid solar cells based on meso-superstructured organometal halide perovskites. Science 2012, 338, 643-647.

[6] Zhang, Q.; Ha, S. T.; Liu, X. F.; Sum, T. C.; Xiong, Q. H. Roomtemperature near-infrared high-Q perovskite whispering-gallery planar nanolasers. Nano Lett. 2014, 14, 5995-6001.

[7] Eaton, S. W.; Lai, M. L.; Gibson, N. A.; Wong, A. B.; Dou, L. T.; Ma, J.; Wang, L. W.; Leone, S. R.; Yang, P. D. Lasing in robust cesium lead halide perovskite nanowires. Proc. Natl. Acad. Sci. USA 2016, 113, 1993-1998.

[8] Fu, Y. P.; Zhu, H. M.; Stoumpos, C. C.; Ding, Q.; Wang, J.; Kanatzidis, M. G.; Zhu, X. Y.; Jin, S. Broad wavelength tunable robust lasing from single-crystal nanowires of cesium lead halide perovskites $\left(\mathrm{CsPbX}_{3}, \mathrm{X}=\mathrm{Cl}, \mathrm{Br}, \mathrm{I}\right)$. ACS Nano 2016, 10, 7963-7972.

[9] Du, W. N.; Zhang, S.; Shi, J.; Chen, J.; Wu, Z. Y.; Mi, Y.; Liu, Z. X.; Li, Y. Z.; Sui, X. Y.; Wang, R. et al. Strong exciton-photon coupling and lasing behavior in all-inorganic $\mathrm{Cs} \mathrm{PbBr}_{3}$ micro/nanowire Fabry-Pérot cavity. ACS Photonics 2018, 5, 2051-2059.

[10] Park, K.; Lee, J. W.; Kim, J. D.; Han, N. S.; Jang, D. M.; Jeong, S.; Park, J.; Song, J. K. Light-matter interactions in cesium lead halide perovskite nanowire lasers. J. Phys. Chem. Lett. 2016, 7, 3703-3710.

[11] Yakunin, S.; Protesescu, L.; Krieg, F.; Bodnarchuk, M. I.; Nedelcu, G.; Humer, M.; De Luca, G.; Fiebig, M.; Heiss, W.; Kovalenko, M. V. Low-threshold amplified spontaneous emission and lasing from colloidal nanocrystals of caesium lead halide perovskites. Nat. Commun. 2015, 6, 8056

[12] Wang, Y.; Li, X. M.; Song, J. Z.; Xiao, L.; Zeng, H. B.; Sun, H. D. All-inorganic colloidal perovskite quantum dots: A new class of lasing materials with favorable characteristics. Adv. Mater. 2015, 27, $7101-7108$.

[13] Xuan, T. T.; Yang, X. F.; Lou, S. Q.; Huang, J. J.; Liu, Y.; Yu, J. B.; Li, H. L.; Wong, K. L.; Wang, C. X.; Wang, J. Highly stable $\mathrm{CsPbBr}_{3}$ quantum dots coated with alkyl phosphate for white light-emitting diodes. Nanoscale 2017, 9, 15286-15290.

[14] Chen, X.; Hu, H. W.; Xia, Z. M.; Gao, W.; Gou, W. Y.; Qu, Y. Q.; $\mathrm{Ma}, \mathrm{Y} . \mathrm{Y} . \mathrm{CsPbBr}_{3}$ perovskite nanocrystals as highly selective and sensitive spectrochemical probes for gaseous $\mathrm{HCl}$ detection. $J$. Mater. Chem. C 2017, 5, 309-313.

[15] Swarnkar, A.; Chulliyil, R.; Ravi, V. K.; Irfanullah, M.; Chowdhury, A.; $\mathrm{Nag}, \mathrm{A}$. Colloidal $\mathrm{CsPBr}_{3}$ perovskite nanocrystals: Luminescence beyond traditional Quantum dots. Angew. Chem., Int. Ed. 2015, 127, 15644-15648.

[16] Stoumpos, C. C.; Malliakas, C. D.; Peters, J. A.; Liu, Z. F.; Sebastian, M.; Im, J.; Chasapis, T. C.; Wibowo, A. C.; Chung, D. Y.; Freeman, A. J. et al. Crystal growth of the perovskite semiconductor $\mathrm{CsPbr}_{3}$ : A new material for high-energy radiation detection. Cryst. Growth Des. 2013, 13, 2722-2727.

[17] Di, X. X.; Hu, Z. M.; Jiang, J. T.; He, M. L.; Zhou, L.; Xiang, W. D.; Liang, X. J. Use of long-term stable $\mathrm{CsPBr}_{3}$ perovskite quantum 
dots in phospho-silicate glass for highly efficient white LEDs. Chem. Commun. 2017, 53, 11068-11071.

[18] Dang, Z. Y.; Shamsi, J.; Palazon, F.; Imran, M.; Akkerman, Q. A.; Park, S.; Bertoni, G.; Prato, O.; Brescia, R.; Manna, L. In situ transmission electron microscopy study of electron beam-induced transformations in colloidal cesium lead halide perovskite nanocrystals. ACS Nano 2017, 11, 2124-2132.

[19] Zhu, H. M.; Fu, Y. P.; Meng, F.; Wu, X. X.; Gong, Z. Z.; Ding, Q.; Gustafsson, M. V.; Trinh, M. T.; Jin, S.; Zhu, X. Y. Lead halide perovskite nanowire lasers with low lasing thresholds and high quality factors. Nat. Mater. 2015, 14, 636-642.

[20] Becker, M. A.; Vaxenburg, R.; Nedelcu, G.; Sercel, P. C.; Shabaev, A.; Mehl, M. J.; Michopoulos, J. G.; Lambrakos, S. G.; Bernstein, N.; Lyons, J. L. et al. Bright triplet excitons in caesium lead halide perovskites. Nature 2018, 553, 189-193.

[21] Rainò, G.; Becker, M. A.; Bodnarchuk, M. I.; Mahrt, R. F.; Kovalenko, M. V.; Stöferle, T. Superfluorescence from lead halide perovskite quantum dot superlattices. Nature 2018, 563, 671-675.

[22] Enderlein, J.; Pampaloni, F. Unified operator approach for deriving Hermite-Gaussian and Laguerre-Gaussian laser modes. J. Opt. Soc. Am. A 2004, 21, 1553-1558.

[23] Zhou, C.; Zhong, Y. C.; Dong, H. X.; Zheng, W. B.; Tan, J. Q.; Jie, Q.; Pan, A. L.; Zhang, L.; Xie, W. Cooperative excitonic quantum ensemble in perovskite-assembly superlattice microcavities. Nat. Commun. 2020, 11, 329.

[24] Fu, J. H.; Xu, Q.; Han, G. F.; Wu, B.; Huan, C. H. A.; Leek, M. L.; Sum, T. C. Hot carrier cooling mechanisms in halide perovskites. Nat. Commun. 2017, 8, 1300.

[25] Fang, H. H.; Adjokatse, S.; Shao, S. Y.; Even, J.; Loi, M. A. Long-lived hot-carrier light emission and large blue shift in formamidinium tin triiodide perovskites. Nat. Commun. 2018, 9, 243.

[26] Jia, Y. F.; Kerner, R. A.; Grede, A. J.; Rand, B. P.; Giebink, N. C. Continuous-wave lasing in an organic-inorganic lead halide perovskite semiconductor. Nat. Photonics 2017, 11, 784-788.

[27] Dang, C.; Lee, J.; Breen, C.; Steckel, J. S.; Coe-Sullivan, S.; Nurmikko, A. Red, green and blue lasing enabled by single-exciton gain in colloidal quantum dot films. Nat. Nanotechnol. 2012, 7 , 335-339.

[28] Klimov, V. I.; Mikhailovsky, A. A.; Xu, S.; Malko, A.; Hollingsworth, J. A.; Leatherdale, C. A.; Eisler, H. J.; Bawendi, M. G. Optical gain and stimulated emission in nanocrystal quantum dots. Science 2000, 290, 314-317.

[29] Yantara, N.; Bhaumik, S.; Yan, F.; Sabba, D.; Dewi, H. A.; Mathews, N.; Boix, P. P.; Demir, H. V.; Mhaisalkar, S. Inorganic halide perovskites for efficient light-emitting diodes. J. Phys. Chem. Lett. 2015, 6, 4360-4364. 